\title{
ACRAL-ACROMA MALIGNANT MELANOMA. A CLINICAL CASE REPORT AND REVIEW OF LITERATURE
}

\author{
CORINA-MARIA BOTICI ${ }^{1}$, LĂCRIMIOARA COVACI ${ }^{2}$, \\ CLAUDIA JUDEA-PUSTA ${ }^{3}$, ANDREI PAȘCALĂU ${ }^{3}$
}

\author{
${ }^{1}$ Faculty of Medicine and Pharmacy, Oradea, Romania \\ ${ }^{2}$ Faculty of Letters, Babeș-Bolyai University, Cluj-Napoca, Romania \\ ${ }^{3}$ Morphological Disciplines Department, Faculty of Medicine and Pharmacy, \\ University of Oradea, Romania
}

\begin{abstract}
Objective. The main objective of this paper is to emphasize the importance of the early diagnosis of certainty in the cases of acral-acroma malignant melanoma in order to increase the survival rate.

Method. The paper is a review of specialty literature and presents a clinical case of acral-acroma malignant melanoma. Clinical case: acral-acroma malignant melanoma with confirmed lymph node metastases and possibly secondary remote dissemination to the skin in a male patient aged 16 years. We conducted histopathological and immunohistochemical examinations of the collected tissue-fragments.

Results. The results obtained from the histochemical and immunohisto-chemical examinations are presented, as well as the problems of differential diagnosis.

Conclusion. The early diagnosis of certainty significantly increases the survival rate. Knowledge and awareness of the population on the use of preventive measures regarding the occurence of the acral-acroma malignant melanoma is therefore required.
\end{abstract}

Keywords: acral-acroma malignant melanoma, lymph node metastases, immunohistochemistry, diagnosis certainty

\section{Introduction}

Malignant melanoma (MM) is the most severe skin neoplasia; it appears in the malignant transformation of melanocytes, cells of neuroectodermal origin that synthesize the melanin pigment as a result of defects in their genome, innate or generated by ultraviolet rays, having the tendency to metastasize [1].

The predominant location of the malignant melanoma is on the skin and mucous membranes, but it can appear anywhere the migrated cells of the neural crest that secrete melanin exist, e.g.: brain, retina, meninges.

The melanocytes have as main function the production of melanin, pigment with UV-absorbing role, protecting the body from their noxious action. Another assigned role of

Manuscript received: 16.04.2017

Received in revised form: 06.06.2017

Accepted: 20.06.2017

Address for correspondence: boticicorina@yahoo.com melanin is the neutralization of the free radicals.

Malignant melanomas show different macromicroscopic particularities, such as:

- Superficial spreading melanoma (70\%): it occurs more frequently in adults over 40 yearsold, is localized on the women's ankle and and on men's back trunk, characterized by the extension in surface of various colors (black, brown, red, white), with irregular borders;

- Nodular melanoma (10-15\%): it occurs more frequently in males, the lesions are dark blue, distinctive edges and a relatively symmetrical shape, rapidly expanding; in medical practice other forms of amelanotic (acroma) nodular melanoma are known, which often pose serious problems with respect to the differential diagnosis from other tumor pathologies;

- Lentiginous Melanoma (10\%): occurs without predilection in both sexes, it is characterized by a large 
lesion $(1-5 \mathrm{~cm})$, flat, especially on the face, neck skin and sun exposed areas;

- Acral melanoma (2-8\% of Caucasians, $40-60 \%$ of Black population, Hispanic and Asian): is located on the skin of the palms, plantar surface and subungvial.

There are also some rare histologic subtypes:

- Melanoma of minimal deviation type (borderline);

- Lentiginous mucosal melanoma (oral, genital);

- Desmoplastic melanoma (a very rare form, approx. $1 \%$ is characterized by perineural invasion);

- Amelanotic (acroma) melanoma poses serious clinical diagnosis problems with the macule and papule [2].

\section{Pathogenesis}

The majority of melanomas occur sporadically, but some are hereditary (5-10\% rates).

The malignant transformation of melanocytes is a process which takes place progressively and involves activating mutations of proto-oncogenes and loss of function mutations of tumor suppressor genes. Up to $40 \%$ of individuals affected by the familial melanoma mutations present in the germline of the CDKN2A, the gene encoding the tumor suppressor p16, an inhibitor of kinases that regulates the G1-S progression through the retinoblastoma protein $(\mathrm{pRb})$.

The somatic activating mutations of the protooncogenes BRAF and NRAF are present in numerous cases of melanoma; in general these mutations, which stimulate the proliferation and the cell survival through the activation of the protein kinase pathway regulated by the extracellural signal (ERK), are mutually exclusive as the BRAF acts downstream of the $\mathrm{pRb}$ gene.

Some melanomas, especially those that occurr in the acral areas or on the mucus membranes, present activating mutations in the tyrosine kinase receptor c-kit. The agents that selectively inhibit BRAF and c-KIT mutations caused significant responses in patients with metastatic tumors with mutated BRAF and c-KIT, which is an encouraging example of the effect of molecular therapy against this daunting disease $[3,6]$.

In the case of inoperable or metastatic melanomas with positive BRAF V600 mutation, Vemurafenib treatment is implemented either as monotherapy or in combination with Dabrafenib; these drugs are inhibitors of the serinethreonine kinase BRAF, inhibiting the tumor type cellular proliferation. [6].

\section{Treatment}

The first choice treatment in the case of the malignant melanoma is represented by the wide excision, with safety margins, of the primary tumor, of the secondary tumor and the ablation of the sentinel lymph nodes.

Another alternative treatment with relatively favorable results is by radiotherapy, chemotherapy and immunotherapy, but it does not present a high efficacy for the types of invasive and metastatic melanomas.

According to recent studies, it is proven that Dacarbazine remains the preferred medication in disseminated melanoma, along with interleukins, biochimiotherapy and interferon, which give encouraging results. [4,7]

\section{Material and method \\ Clinical case}

We will describe a case of acral-acroma malignant melanoma with confirmed lymph node metastases and possibly secondary remote dissemination to the skin in a patient aged 16 years.

The male patient presented in August 2016 at the emergency department with: bleeding and swelling of the left hallux, growth of the toe volume, and a left groin tumor formation (patient could not specify the exact time of its appearance or changes).

Examination revealed the following: left toe thickened and deformed, dystrophic nail and 2-3 mm aberrant on an area where the skin around presented a blackish-brown ulceration; $10 / 8 \mathrm{~cm}$ purple tumor formation, tough, welldefined, relatively homogeneous, inguinofemoral location, possibly related to the inguinal lymph nodes, which were firm, tough, and sized up to $1-1.5 \mathrm{~cm}$.

The patient was investigated hematologically, biochemically (normal LDH) and by imaging for other determinations, but tests were negative with the exception of the homolateral inguinal lymph node presence.

Considering these data, the sampling of a bioptic specimen of the presumptive primary tumor (hallux) was performed and a ganglional dissection was attempted from the left groin, but the extension of the lymphadenopathic block did not allow the execution in optimum time and a lymphonodular formation sample was taken instead.

Two pieces were sent to the Pathology Department with the clinical diagnosis of left inguinal lymphadenopathic block, bioptic TU fragment of the left toe nail bed:

A. Lymph node $2 / 1.5 / 1 \mathrm{~cm}$, gray colored.

B. Two pieces of $0.5 \mathrm{~cm}$ and $0.1 \mathrm{~cm}$, gray coloured.

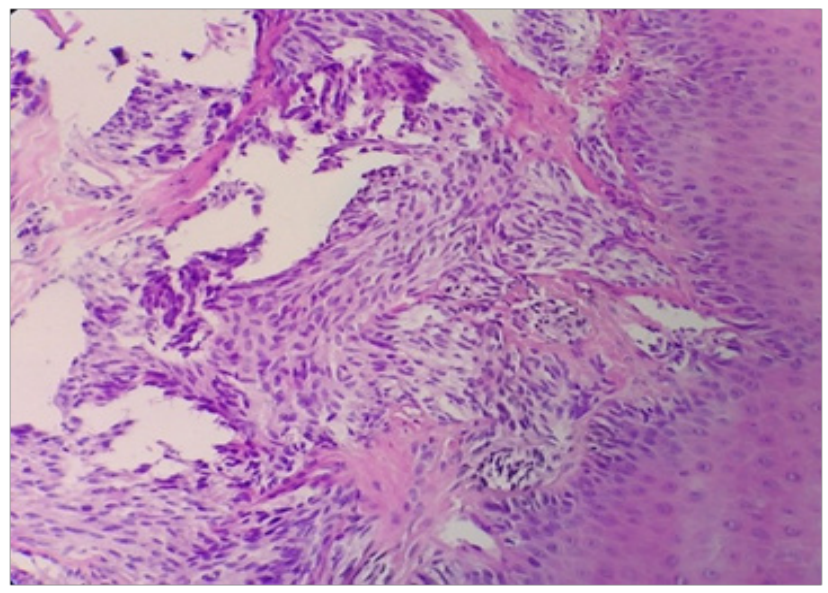

Figure 1. HE 20X. 
The pieces analyzed microscopically (B) show a papilliferous hyperkeratotic epidermis and at the level of the superficial dermis we can observe a proliferation of spindle cells, with round or oval nuclei, some tachicroms and others with prominent nucleoli, the cytoplasm easily eosinophilic, organized in swirls or fasciculated, storiform, with intense mitotic activity. (Figure 1)

At the level of the lymph node (A.) examined microscopically we observe: wide cellular area with the characteristics described above, rare remaining lymphoid follicles (Figures 2 and 3), rare medium sized cells with brown intracellular pigment (Figure 4).

The microscopic aspects described above did not allow the enunciation of higly specific diagnosis, in this regard complementary examinations being necessary, and therefore the diagnostic on phases was considered: a fusiform cell proliferation with features of malignancy and lymphnode secondary determinations. It further recommends the performance of complementary immunohistochemical examinations for a diagnostic of certainty, the determination of the histogenetic origin, and specialized treatment.

The immunohistochemical analysis revealed the existance of malignant melanoma metastasis at the level of the lymph node S100 + (Figure 5), MART1 + (Figure 6) corresponding to the immune profile of the biopsy piece taken from the hallux the S100 + (Figure 7) MART1 + (Figure 8). The presence of the BRAF gene was also evaluated and considered negative. (Figure 9).

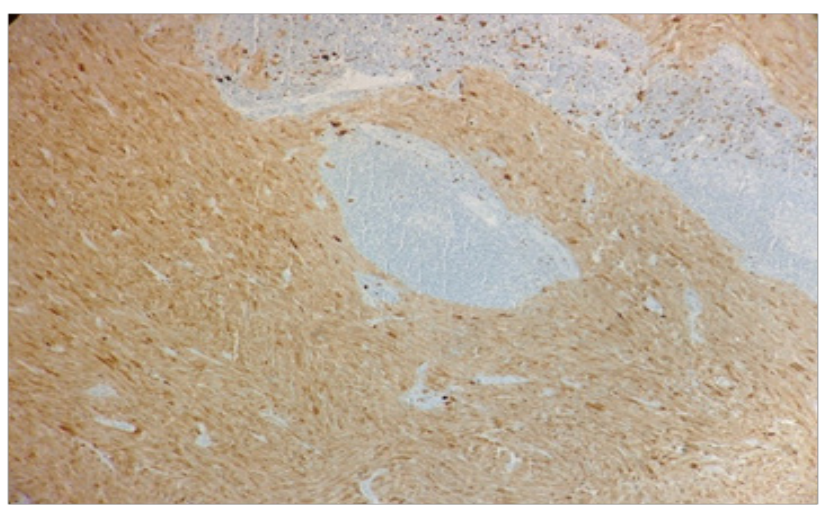

Figure 5. S100+, 10X.

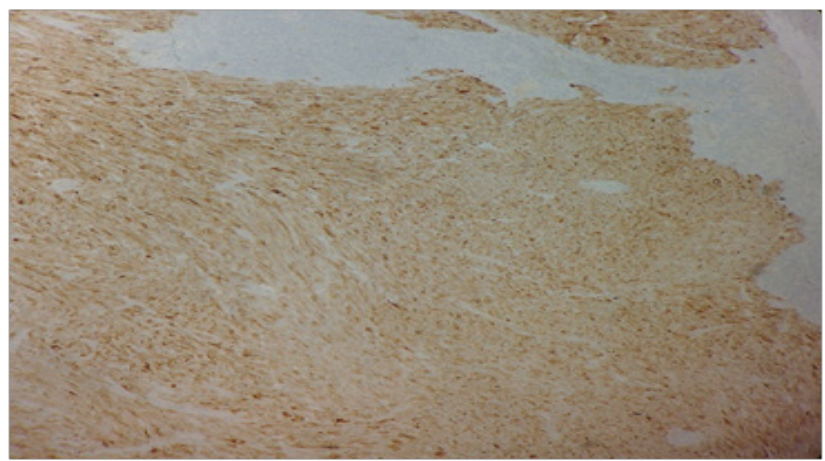

Figure 6. MART1+, 10X.

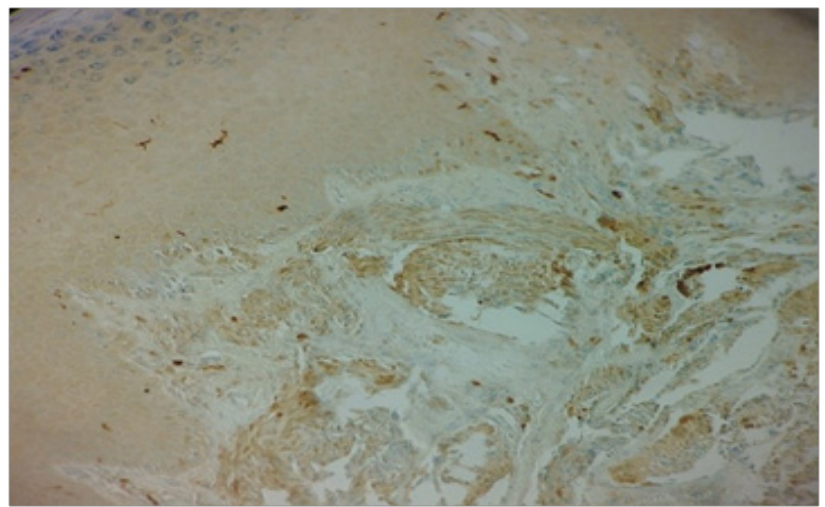

Figure 7. S100+, 10X.

Figure 4. HE 40X. 


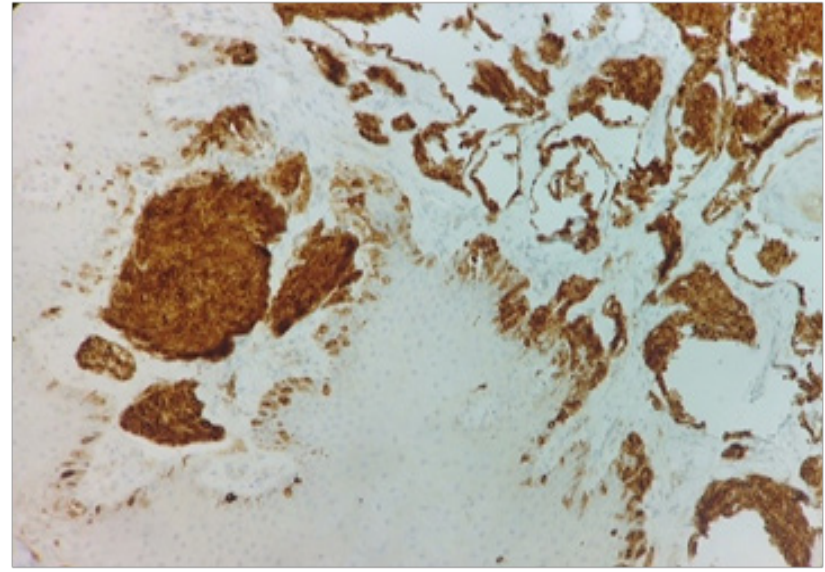

Figure 8. MART1+, 10X.

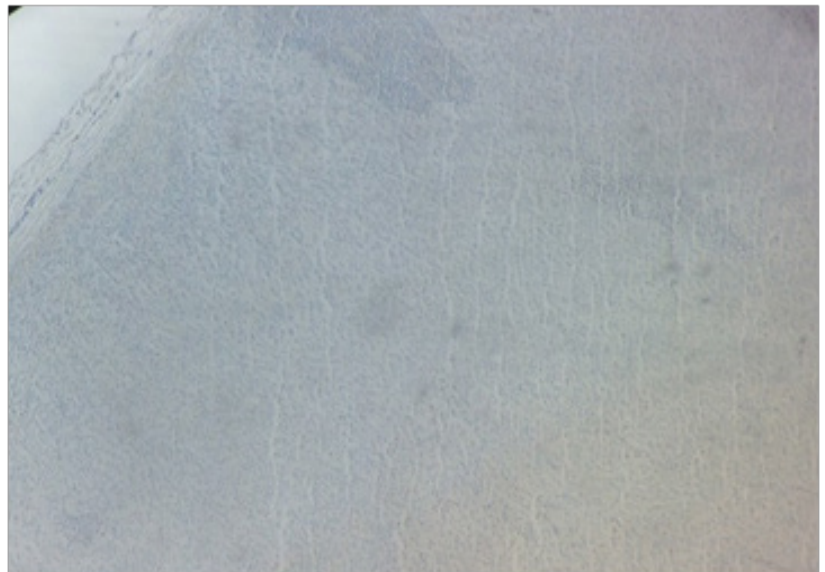

Figure 9. BRAF neg., 10X.

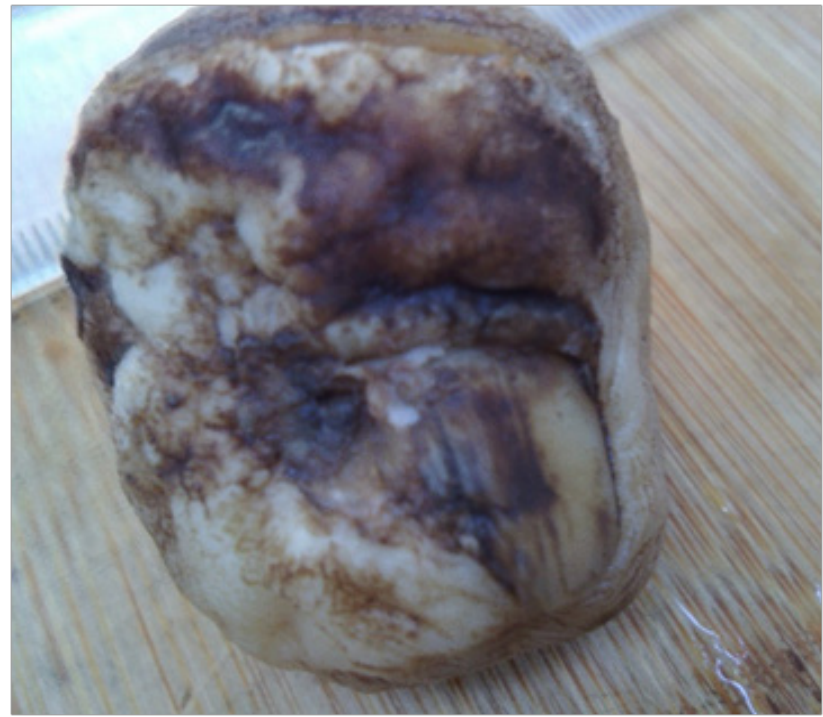

Figure 10. Left toe, spare part from amputation surgery, on which we witness a shallow extension of the primary tumor and ulcerated area.

The recommended treatment in this case: the larger excision of the primary tumor and of the skin and the left inguinal nodal ablation, which will be continued with the following adjuvant therapy: radioteraphy (if necessary), chemotherapy and immunotherapy $[5,6]$.

\section{Discussion}

According to data from literature, the malignant melanoma has a low incidence in children (approx. $0.5 \%$ of all melanomas). At the same time, the scarcity of acral type melanomas can be easily noticed, as well as problems of differential diagnosis imposed by the amelanotic (acroma) nodular melanoma vs. other skin tumors.

Considering these aspects, we can intuit the need for a high medical vigilance and an increased attention of the medical specialist so that any case which includes at least one of the criteria mentioned above will be of particular interest.

The frequency of the occurance and the death rate in the case of the malignant melanoma is steadily increasing, unacceptably high if we were to take into account the fact that this pathology can be diagnosed, in most cases, in a stage in which the cure is possible.

Consequently, it is important to draw the attention towards the medical vigilence with respect to the malignant melanoma and towards the use of the prevention methods regarding the occurrence of this cruel pathology.

\section{Conclusions}

The frequency of the malignant melanoma is increasing predominantly in white Caucasians, with high incidence lately due to the excessive exposure to UV-B radiation (UVR-b).

Recent studies of deep sequencing have confirmed 
that the tumoral genome comprises thousands of acquired mutations, the majority being induced by UV rays, which marks the significance of the immunohistochemical phenotyping towards the detection of the mutated BRAF gene, so that when it is present the treatment can be augmented and the survival rate significantly increases to 5 years.

Moreover, the early diagnosis certainty significantly modifies the survival rate, increasing it, a fact recognized by the American Cancer Society, as the prognosis appears more favorable among younger age groups.

Finally, an increased medical vigilance and a broader awareness and public outreach can bring considerable improvements to the identification of the diagnosis and the proper treatment of these lesions, definitely increasing the survival rate.

\section{References}

1. Liu ZJ, Herlyn M. Melanoma. In: DeVita VT Jr, Hellman S,
Rosenberg SA, eds. Cancer: Principles and Practice of Oncology. 7th ed. Philadelphia: Lippincott, Williams \& Wilkins, 2005, pp1745-1824.

2. Corona R, Mele A, Amini M, De Rosa G, Coppola G, Piccardi $\mathrm{P}$, et al. Interobserver variability on the histopathologic diagnosis of cutaneous melanoma and other pigmented skin lesions. J Clin Oncol. 1996;14(4):1218-1223.

3. Kumar V, .Abbas AK,Aster JC. Robbins Patologie, Bazele morfologice si fiziopatologice ale bolilor [Robbins Pathological Basis of Disease], Ed. a IX-a , Editura Medicală Callistro, 2015; pp. 867

4. Rosai and Ackerman's Surgical Pathology, Rosai, 10th ed, 2011. [Online]

5. Vennepureddy A, Thumallapally N, Motilal Nehru V, Atallah JP, Terjanian T. Novel Drugs and Combination Therapies for the Treatment of Metastatic Melanoma. J Clin Med Res. 2016;8(2):63-75.

6. Queirolo P, Spagnolo F. BRAF plus MEK-targeted drugs: a new standard of treatment for BRAF-mutant advanced melanoma. Cancer Metastasis Rev. 2017;36(1):35-42.

7. Sternberg's Diagnostic Surgical Pathology, 2015. [Online] 